\title{
Parental Death, Grief and Bereavement: A Qualitative Study of College Students Who Have a Parent with Cancer
}

\author{
Yong Tang ${ }^{1} \&$ Yingying $\mathrm{Li}^{1}$ \\ ${ }^{1}$ Sociology Department, Shenzhen University, China \\ Correspondence: Yong Tang. E-mail: tangyong@szu.edu.cn
}

Received: June 8, 2019 Accepted: June 28, $2019 \quad$ Online Published: July 23, 2019

doi:10.5539/ass.v15n8p56 URL: https://doi.org/10.5539/ass.v15n8p56

\begin{abstract}
Objective The loss of a parent with cancer is one of the most traumatic events a college student can face. The purpose of this study was to identify the main elements of bereavement experiences among college students in China who lost a parent with cancer. Methods A total of 18 college students volunteered to take part in three focus groups. Results Four themes were developed through content analysis: facing huge life's changes, experiencing loss and suffering, challenges and coping strategies for facing parental death, and re-interpreting death. These four main themes were then divided into 15 sub-themes. Conclusions The analysis showed that parental death may negatively influence college students' bereavement processes. Bereavement should include screening to aid in the recognition of high-risk adolescents through screening survey and screening interviews.
\end{abstract}

Keywords: parental death, grief, bereavement, college student, cancer

\section{Background}

Approximately $1 \%$ of college students in China experience parental death during their college phase (Zhang, 2015; Schooling et al., 2011). Research has found that parental death increases college students' short and long-term risks for mental health problems (Sandler et al., 2003; Currier, Neimeyer, \& Berman, 2008). Given these negative outcomes (Hooyman \& Kramer, 2013; Stroebe, Hansson, Stroebe, \& Schut, 2001), the development of effective preventive interventions has high public health significance (Parkes, Laungani, \& Young, 1997; Parks \& Prigerson, 2013). The grief experienced by college students have received growing attention among researchers (Frank, 2008; Roberts, 2016). The expanding body of literature on this topic has resulted in greater awareness among scholars and practitioners about the emotional challenges confronted by college students. More specifically, scholars have begun to ascertain that parental death, grief and bereavement experienced by college students play a more pronounced role in their well-being than has been previously realized by research.

Cancer is a major cause of disability and mortality in Chinese. The diagnosis and treatment of cancer have resulted in greater caregiving burden being placed on the family. The death of a parent with cancer is one of the most traumatic events that a college student can experience; it increases their risks for depression, anxiety, and hopelessness. Following the death of a parent, college students will generally go through a period of bereavement. During this period of time, they may experience periods of sadness and intense grief. However, some college students will continue to have difficulty adjusting to the loss, which has been defined as the long term emotional hardships following the death of a loved one.

In this study, the author examined grief and bereavement among college students following the death of a parent with cancer. This study presents findings from focus groups. The aim of the current study was to develop a better understanding of what bereaved college students would advise others to do in the same situation. Qualitative interviews were conducted with bereaved college students within the first year after a parent's death. Through systematic analyses of subjective and experiential data, the experiences were categorized based on the thematic content. These data might inform future intervention research and enhance the capacity of health care providers to effectively and appropriately support bereaved college students. Grief counseling is designed to prevent negative outcomes by changing multiple empirically supported risks and protective factors. This intervention might improve the outcomes for bereaved adolescents who have faced parental death. 


\subsection{Symptoms, Grief, and Bereavement of College Students}

Few studies have examined the impact of the death of a parent with cancer during the college years. Balk (2001) found that 22 to $30 \%$ of the college students he studied were in the first year of bereavement following the death of a family member. Grief comprises past, present, and future losses (Kübler-Ross \& Kessler, 2014). Bereavement has been described as a major life crisis with potentially severe effects on the survivor's physical and psychological well-being (Parkes \& Prigerson, 2013). Chinese perceptions of and reactions toward death and dying are strongly associated with the philosophical and religious thought of Confucianism, Buddhism, and Taoism (Li \& Li, 2017; Mak, 2017). Although Chinese traditional thought emphasizes the importance of death, most Chinese, like people of many other cultures, generally view death as misfortune.

\subsection{Chinese Culture and Parental Death with Cancer Among College Students}

Given the uniqueness of Chinese cultural attitudes and beliefs toward death and well as the influences of these beliefs on other Asian countries, research on parental death, grief and bereavement among college students in China can contribute to cross-cultural knowledge in this field. Although several reviews have examined survivors of traumatic deaths, there is limited evidence on this issue, and little empirical support has been provided to bereaved college students facing parental death in China (Chan et al., 2005; Chen \& An, 2009; Zhang, 2011).

\subsection{Framework}

A framework approach to qualitative data analysis was adopted. A framework approach uses a theme-based approach for analysis, reducing data through summarization and synthesis while retaining links to the original data. Using this framework, the author examined the experiences of college students in terms of what they experienced upon losing their parent with cancer. The author used this framework to guide the study, from the development of the interview guide to the interpretation of results (see Figure 1).

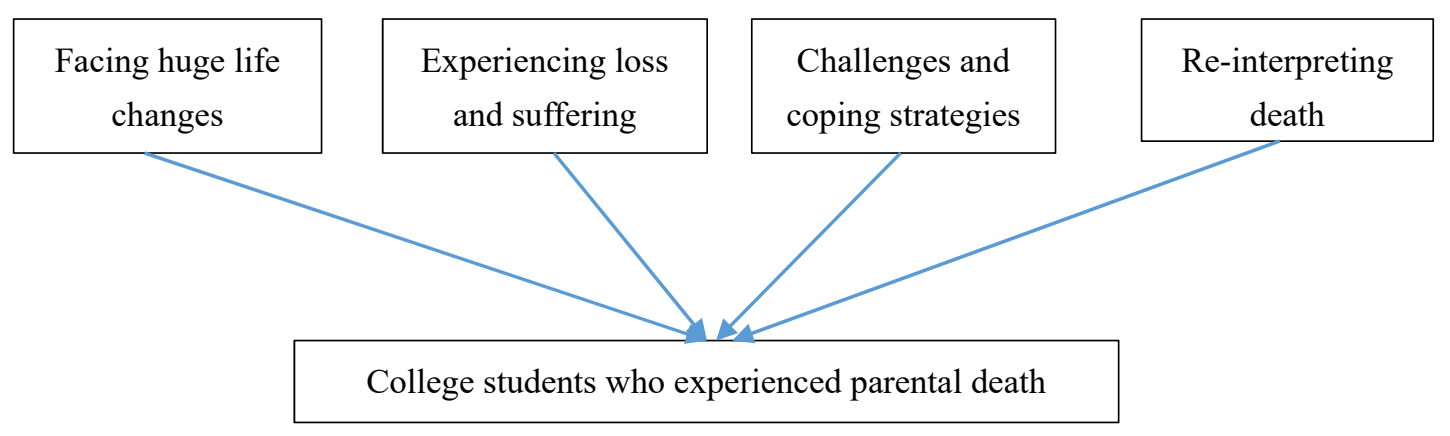

Figure 1. Framework

\section{Methods}

A qualitative approach was adopted using thematic analysis of semi-structured focus group interviews. Eighteen participants were recruited through voluntary bereavement support groups identified by ** University. The qualitative data presented here were collected 12 months following each college student's father's or mother's death. The study was approved by the Research Ethics Committee of ** University. Data were gathered between September 2017 and November 2017. All interviews were recorded and transcribed verbatim by two postgraduates to facilitate the analysis.

Referred individuals who met the eligibility criteria as assessed by telephone were invited to participate in the focus groups. Eligibility criteria were the following: (1) college students who were between 18 and 25 years-old, (2) death of a biological parent with cancer and (3) death that occurred at least 12 months prior. The sample was chosen for two reasons. First, bereaved college students are likely to have considered the possibility of their beloved father's or mother's death. Second, bereaved college students were interviewed because they could reflect on their experiences and feelings after 12 months. Interviewing bereaved college students allowed the author to obtain broader insights on preparedness.

A focus group methodology seemed appropriate because the interactions among bereaved adolescents may facilitate their expression of their experience through discussion in the group (Morrison \& Peoples, 1999).

A total of 18 college students volunteered to take part in the focus groups. Three focus group interviews were conducted. Each group consisted of six participants, all of whom were relatives of an individual lost. The timing of parental death occurred between 2011 and 2016 (Table 1). The major themes identified in the interviews are 
defined in Table 2. There were six first- and second-year undergraduates, six second- and third-year undergraduates, six third-year undergraduates. College students were asked about their understanding of the concepts of death, loss and bereavement.

The interview schedule was designed to yield exploratory data, which were developed by the author. The author conducted the focus group with the assistance of two MSW (Master of Social Work) students. Three sessions were conducted in the focus group. Specific content included (1) challenges since the father/mother died, and (2) coping strategies for parental death. The participants completed a short demographic questionnaire before they started the focus groups. Each focus group was conducted in a classroom and lasted approximately one and a half hours.

The 18 participants in the focused group sessions were asked two open-ended questions: (1) Can you tell me about your challenges after you lost your father/mother? and (2) Can you tell me about your experiences and feelings regarding losing your father/mother?

The process of sampling allowed saturation to be reached. Eighteen participants were sufficient to conclude that no new information or new themes were likely to emerge from further data analysis.

\section{Data Analysis}

The data were analyzed using a thematic analysis approach (Corbin, Strauss, \& Strauss, 2014). The author read all transcripts, making note of verbatim quotes to generate a list of preliminary codes. The author refined the coding structure by adding and modifying codes as new insights emerged. Codes were then sorted into categories that were grouped into broader themes. The themes identified by the author and by the independent reviewer were compared, and agreement was achieved across all major themes (see Table 1 and Table 2).

Table 1. Backgrounds of adolescents facing parental death

\begin{tabular}{cccc}
\hline Interviewee & Time since death & Relationship with the college student & Age of adolescent \\
\hline F1 & 14 months & Mother & 21 \\
F2 & 2 years & Mother & 22 \\
F3 & 15 months & Father & 20 \\
F4 & 18 months & Father & 19 \\
F5 & 16 months & Mother & 20 \\
F6 & 16 months & Father & 22 \\
F7 & 2 years & Father & 21 \\
F8 & 19 months & Mother & 22 \\
F9 & 17 months & Father & 21 \\
F10 & 13 months & Father & 19 \\
M1 & 14 months & Father & 19 \\
M2 & 15 months & Mother & 20 \\
M3 & 2 years & Mother & 22 \\
M4 & 16 months & Father & 23 \\
M5 & 18 months & Father & 18 \\
M6 & 17 months & Father & 19 \\
M7 & 18 months & Mother & 20 \\
M8 & 15 months & Mother & 21
\end{tabular}

* "F" or "M" next to a respondent's number indicates that the participant was female or male, respectively.

Table 2. Example of Data Analysis Process

\begin{tabular}{ll}
\hline Example of Quotation/Meaning Unit & Theme(s) \\
\hline It was really a sad experience for me. I couldn't believe it until two months later. & Experiencing loss and suffering \\
Letting go. Watching and not truly believing what was happening to my loved one. & \\
\hline
\end{tabular}

\section{Results}

The first major theme was labeled "facing life's huge changes". The second major theme was "experiencing loss and suffering". The third major theme was "challenges and coping strategies for facing parental death". The fourth major theme was "re-interpreting death". These four main themes were then divided into 15 sub-themes 
(Table 3), each of which is outlined below with direct quotations from the text. These themes are discussed with selected quotes to illustrate college students' experiences when facing parental death. Many of the issues discussed elicited similar comments and concerns from both undergraduates and postgraduates.

Table 3. Thematic Analysis

\begin{tabular}{ll}
\hline Major Themes & Sub-themes \\
\hline Facing Huge Life Changes & $\begin{array}{l}\text { Experiencing negative reactions; Denying and avoiding death; Sudden changes } \\
\text { in life order. }\end{array}$ \\
Experiencing Loss and Suffering & $\begin{array}{l}\text { Accepting death; Being a son or daughter; Being unfilial to the dead parent } \\
\text { with cancer; Strong feelings of missing. }\end{array}$ \\
$\begin{array}{l}\text { Challenges and Coping Strategies for } \\
\text { Facing Parental Death }\end{array}$ & $\begin{array}{l}\text { Bereavement loneliness; A lack of instrumental social supports; A lack of } \\
\text { emotional social supports; Coping with parental death. } \\
\text { Re-Interpreting Death }\end{array}$ \\
& $\begin{array}{l}\text { The color of death; The rationalization of death; Establishing death; } \\
\text { Transformation and death. }\end{array}$ \\
\hline
\end{tabular}

\section{Theme 1: Facing Huge Life Changes}

Theme 1, "facing huge life changes" included experiencing negative reactions, denying and avoiding death, and sudden changes in life order. The students expressed that longing and sadness were the greatest barriers they faced. Most of them had experienced significant declines in their levels of depressive symptoms. A large majority of them reported having negative reactions to birthdays, holidays, and anniversaries of the death. A minority of them reported being aware of bereavement-related services. Germain, Mayland \& Jack (2016) advocated using such negative reactions as healthy opportunities to express feelings about the death.

\section{Subtheme1: Experiencing Negative Reactions}

Many of the college students were consciously aware of the emotional losses and sense of grief they carried with them. They believed that such loss and grief surpassed other issues as the greatest obstacle they had faced up to that point on their loss journey.

It was really a sad experience for me. I couldn't believe it until two months later. Watching and not truly believing what was happening to my loved one. (M2)

Subtheme 2: Denying and Avoiding Death

College students responded to death with a desire to deny the death, with a willingness to avoid suffering, or with a need to escape the people and events associated with the parental death

The biggest barrier I faced was that one day my father had really passed away. I lost my beloved father. I always miss the days we lived together. I missed him during the Spring Festival, and I always remembered something related to him. (M4)

Subtheme 3: Sudden Changes in Life Order

The death of a parent with cancer created feelings of vulnerably in the college students (Gertler, Levine \& Ames, 2004). They expressed that they faced a significant change in their life order. Whenever a parent with cancer died, there was a process that each healthy family underwent in response to the loss of one of its own. One interviewee said that his father remarried after his mother's death. This process occurred because the family needed to get itself back into a rhythm and restore the balance that was lost when the family member died.

I had to handle a lot of things like an adult. I realized and faced that it was truly a long goodbye and accepted the total change in our lives. Actually, I have never heard of bereavement services. (M5)

\section{Theme 2: Experiencing Loss and Suffering}

Theme 2 "experiencing loss and suffering" included accepting death, being unfilial to the dead parent with cancer, and strong feelings of missing. Of the college students who lived a normal life span, most could not escape the experience of losing a close relative. Experiences of loss and suffering were considered by the college students to be unique human experiences.

\section{Subtheme 1: Accepting Death}

The death of a parent with cancer left the college students feeling empty and alone. This process made it hard to face their daily responsibilities and find happiness in their life. Although it was difficult to forget the impact the lost parent with cancer had on their life, they also had to cope with the death and move towards acceptance. 
I felt that I was the only one left in the world, and that great emptiness and loneliness surrounded me. I had to force myself to accept my father's death (F9).

Subtheme 2: Being Unfilial to the Dead Parent with Cancer

According to Confucianism, the dutiful child's most important responsibility in life is to serve their parents well including treating their memory in an appropriate way after they die and keeping alive the memory of a dead parent through regular rituals (Ho \& Brotherson, 2007).

The son plays a particularly important role in the death rituals. In the Chinese culture, it is important to have family around the individual at the moment of death (Chan et al., 2005). Furthermore, it is particularly important to have the son present at the time of his father's death. If the son is not present, the death of the father can result in the feelings of tremendous guilt for those who are not present at the time of death (Chan et al., 2005).

I wasn't filial to my dying father, because I didn't have the ability to live independently, and I couldn't let my mother live a good life. (M4)

Subtheme 3: Strong Feelings of Missing

A vulnerable parent might become excessively dependent on the child in an effort to replace the lost relationship and intimacy. A majority of the college students expressed their strong feelings of missing their dead parent with cancer.

I always had the illusion that my mother would live forever. (M8)

\section{Theme 3: Challenges and Coping Strategies for Facing Parental Death}

Theme 3 "challenges and coping strategies facing parental death" included bereavement loneliness, a lack of instrumental social supports, a lack of emotional social supports, and coping with parental death. Social support can be found in a number of different places: through friends, family, or support groups. At the same time, they also used coping strategies after the death of their parent with cancer.

\section{Subtheme 1: Bereavement Loneliness}

In China, people are always expected to suppress their feelings to save face. Mourners might instead complain of physical problems such as somatic manifestations of grief. Total emotional release is also not helpful, because Chinese clients might experience catharsis as an immature loss of control

I didn't want my teachers and classmates to know what happened in my family. I cried for a long time when I was alone. (M3)

\section{Subtheme 2: A Lack of Instrumental Social Support}

Bereaved college students lacked instrumental and emotional social supports, which they experienced as barriers for facing parental death. Instrumental social support provides assistance in a tangible way. Some studies have shown that parental income has an important positive effect on educational attainment (Chevalier et al., 2013; Noble et al., 2014), and that achievement in school is positively linked to parental investments of time (Benner, Boyle, \& Sadler, 2016; Dufur, Parcel, \& Troutman, 2013). In the financial realm, the following quotes were representative of responses regarding the greatest barrier.

Money became strained after my father's death. Both my sister and I were in college, and we still needed financial support from our family. The money strain made our living costs much harder. (M4)

Subtheme 3: A Lack of Emotional Social Support

A lack of emotional social support might be difficult for college students who have faced parental death. For example, emotional social support to college students might involve listening and offering sympathy after they receive bad news.

I didn't have many relatives in Shenzhen. Most of my family members were in Hunan province. After my mother's funeral, I returned directly to school. Only a few friends in college knew that my mother died. I often felt lonely. (M3)

Subtheme 4: Coping with Parental Death

Coping proved to be a useful and effective way for college students to adjust to the death of a parent with cancer. The particular successfulness of a coping strategy depends on the benefits of giving and receiving help from others facing the same crisis. In this study, some college students had to find a new role. With another meaningful role, coping strategies afforded the opportunity for the college student to reinvest energy and love in another valued pursuit. Some were involved in self-help groups and were affected by participation in the group. 
I put more energy into my ordinary studies. Through study achievements and progress, I lightened the grief of losing my mother. (M2)

\section{Theme 4: Re-Interpreting Death}

Theme 4, "re-interpreting death", included the color of death, the rationalization of death, establishing death, and transformation and death. Parental death led the college students to search for the meaning of death, with the goal of integrating the loss through meaning reconstruction processes, such as interpreting death through color, rationalizing death, establishing the meaning of death, and transformation and growth.

\section{Sub-Theme 1: The Color of Death}

The Chinese word for "color" is yánsè. In classical Chinese, color refers to certain values and more accurately indicates "emotion". White symbolizes brightness, purity, and fulfillment. White is also the color of mourning after death, is associated with death and is used predominantly in funerals. Chinese college students who lost their parent with cancer stated that they wore white clothes to the funeral. They also expressed that red was another color that was traditionally used in funerals.

I drew a picture in blue. Blue is a light color. When my father died, his body became lighter and lighter, and it seemed to be flying overhead. Death is a natural thing. Everyone will die someday. (F8)

I think black is the death color. Death was a horrible thing. When my father died, we had to wear black gauze during the funerals. Death is an unknown thing. None of us knew what the death world was. (F2)

Subtheme 1: Rationalization of Death

College students rationalized death because they did not think it could be avoided. However, death is horrible; it thwarts many desires.

My father no longer suffers from cancer pain. It should be a relief for him. (M6)

\section{Subtheme 2: Establishing the Meaning of Death}

Confucianism is one of the main philosophies in the Chinese culture. It influences Chinese beliefs on death and dying. Confucianism provides a moral basis for filial piety (duty to parents), which is central to death and dying. Confucians believe in a supernatural heaven and believe in the continued existence by which the soul extends Chinese duty after death in the form of ancestor worship. When the parents were dying, the children were expected to care for them out of filial piety. Death, according to Confucian principles, is good when it is meaningful, particularly when one dies for the preservation of Confucian virtues (Chan \& Chow, 2006).

When I was very young, my mother always told me that we should live a good life and try to think of others. Although my mother died, but her life was complete. (F8)

\section{Discussion}

The transition from a teenager into an adult is already a confusing time; thus, losing a parent with cancer in the midst of everything going on in college is devastating, because it can sometimes feel like you need to do it all on your own. In this study, the author conducted focus groups with adolescents who lost their parents with cancer to identify four major stages associated with bereavement experiences that significantly negatively influenced the bereavement process. The study had four findings: college students had prolonged grief during the bereavement process; they rarely share the pain of bereavement with families, and they would face challenges after parental death.

\subsection{Prolonged Grief During the Bereavement Process in College Students}

College students experienced low mood, a loss of interest in activities, and avoidance of reminders of the loss. It remained unclear whether the college students who suffered greatly following the loss of a beloved parent experienced symptoms due to the death, whether they began to experience these symptoms before the death, or whether grief was a proxy for prior depression (Xu et al., 2015; Xiu et al., 2017). Notably, depressive disorder and complicated grief frequently co-existed among college students who faced parental death. Some of the interviewees had no obvious grief response at the beginning of the bereavement process, usually because they concealed their expressing their grief.

\subsection{The Pain of Bereavement Was Rarely Shared with Their Families}

This study found that college students rarely sought supports from their families. Some of the interviewees reported that they were unwilling to share the pain of bereavement with their families. On the one hand, they were seeking independence, and on the other hand, they were affected by Chinese cultural values, i.e., death is a 
taboo topic and is often avoided in conversation. These cultural factors prevented them from expressing their grief.

\subsection{College Students Faced Challenges After the Death of a Parent with Cancer}

After the death of a parent with cancer, college students faced huge life changes, such as, negative reactions and changes in life order. The surviving parent spent less time helping college students with their education and providing parental supervision than two parents would; thus, the marginal benefits of the college student's education decreased.

The loss of a parent with cancer is tremendously traumatic and may affect bereaved college students' emotional status and values. The trauma of bereavement may make it difficult for college students to study, and they may withdraw temporarily or permanently from college. When a parent with cancer disappears from the household, college students may no longer have the motivation to continue their education.

\subsection{Lack of Death Education and Grief Counseling in China}

From the respondents' interviews, talking about death remains a taboo in China. The point of providing education on death-related issues is to tell college students about the meaning of death and to encourage them to extend the length of their life despite losing their parent with cancer. China continues to lack death education. Most of the college students who lost parents relied on themselves, and few of them would turn to grief counselors for help. The way a counselor responds to grieving college students may have a profound impact on students' future lives. Grief counselors can provide companions for bereaved college students.

\section{Conclusion}

The current study shows that struggling with a loved one's death to attempt to understand and see something good arising from the struggle is important in laying the foundation for the bereavement of college students. It may be that the willingness or ability to engage in this work is associated with belief systems, or religious attitudes that the bereaved college students had before the event of bereavement (Taku et al., 2008). Future research would benefit from the identification of stable factors that might facilitate engagement in rumination soon after bereavement.

To date, little is known about how college students' grief manifests over time and how other indicators of emotional well-being might be affected. Currently, there is a rich body of literature on interventions for dementia caregivers that cover important issues such as stress, burden, depression, and coping. However, with a few notable exceptions, there is virtually no published research focusing on the efficacy of pre-death loss and grief interventions for college students, and there is an absence of research exploring the relationship between post-intervention college students' grief and bereavement over time.

This study adds to the body of bereavement knowledge in the Chinese context and elucidates college students' bereavement experience. The findings of this study may have implications for school social workers and clinical practice. School social workers should identify the spiritual, psychological, and physical needs of college students and attempt to provide culturally oriented bereavement counseling. The present study has several limitations. Despite the limitations, the present study could be extended to Chinese samples to explore how they cope with death and dying.

\section{Declarations}

\section{Ethics Approval}

This study was conducted with the approval of the Human Resources and Ethics Committee Approval of Shenzhen University (Ref. 2018042).

\section{Interviewees' Consent}

Written consent obtained.

\section{Availability of data and material}

All data generated or analyzed during this study are included in this published article.

\section{Acknowledgment}

The work described in this paper was fully supported by a special fund for "climbing plan" from cultivation of scientific and technological innovation of university students from Guangdong, China (No. pdjhb0442). I would like to acknowledge the efforts of Yilin Chen and Tingting Long who assisted in performing the interviews. 


\section{References}

Balk, D. E. (2001). College student bereavement, scholarship, and the university: A call for university engagement. Death Studies, 25(1), 67-84. https://doi.org/10.1080/07481180126146

Benner, A. D., Boyle, A. E., \& Sadler, S. (2016). Parental involvement and adolescents' educational success: The roles of prior achievement and socioeconomic status. Journal of Youth and Adolescence, 45(6), 1053-1064. https://doi.org/10.1177/0004944118764684

Berg, L., Rostila, M., \& Hjern, A. (2016). Parental death during childhood and depression in young adults-a national cohort study. Journal of Child Psychology and Psychiatry, 57(9), 1092-1098. https://doi.org/10.1111/jcpp.12560

Chan, C. L., Chow, A. Y., Ho, S. M., Tsui, Y. K., Tin, A. F., Koo, B. W., \& Koo, E. W. (2005). The Experience of Chinese Bereaved Persons: A Preliminary Study of Meaning Making and Continuing Bonds. Death Studies, 29(10). https://doi.org/10.1080/07481180500299287

Chen, S. G., \& An, X. L. (2009). Attitudes of death from 436 university students. Journal of Chinese Psychological Study, 23(4), 303-304 (in Chinese).

Chevalier, A., Harmon, C., O'Sullivan, V., \& Walker, I. (2013). The impact of parental income and education on the schooling of their children. IZA Journal of Labor Economics, 2(1), 8. https://doi.org/10.1186/2193-8997-2-8

Clarke, V., \& Braun, V. (2014). Thematic analysis. In Encyclopedia of critical psychology (pp. 1947-1952). Springer New York.

Corbin, J., Strauss, A., \& Strauss, A. L. (2014). Basics of qualitative research. Sage.

Currier, J. M., Neimeyer, R. A., \& Berman, J. S. (2008). The effectiveness of psychotherapeutic interventions for bereaved persons: A comprehensive quantitative review. Psychological Bulletin, 134(5), 648. http://dx.doi.org/10.1037/0033-2909.134.5.648

Dufur, M. J., Parcel, T. L., \& Troutman, K. P. (2013). Does capital at home matter more than capital at school? Social capital effects on academic achievement. Research in Social Stratification and Mobility, 31, 1-21. https://doi.org/10.1016/j.rssm.2012.08.002

Frank, J. B. (2008). Evidence for grief as the major barrier faced by Alzheimer caregivers: A qualitative analysis. American Journal of Alzheimer's Disease \& Other Dementias, 22(6), 516-527. https://doi.org/10.1177/1533317507307787.

Germain, A., Mayland, C. R., \& Jack, B. A. (2016). The potential therapeutic value for bereaved relatives participating in research: An exploratory study. Palliative \& Supportive Care, 14(5), 479-487. https://doi.org/10.1017/S1478951515001194.

Gertler, P., Levine, D. I., \& Ames, M. (2004). Schooling and parental death. Review of Economics and Statistics, 86(1), 211-225.

Hooyman, N. R., \& Kramer, B. J. (2013). Living through Loss: Interventions across the Life Span. Columbia University Press.

Ho, S. W., \& Brotherson, S. E. (2007). Cultural influences on parental bereavement in Chinese families. OMEGA-Journal of Death and Dying, 55(1), 1-25. https://doi.org/10.2190/4293-202L-5475-2161

Kang, K. A. (2010). Comparison of meaning in life and death attitude between participants and non-participants in well-dying education. Journal of Korean Oncology Nursing, 10(2), 156-162. https://doi.org/10.5388/jkon.2010.10.2.156

Kübler-Ross, E., \& Kessler, D. (2014). On grief and grieving: Finding the meaning of grief through the five stages of loss. Simon and Schuster.

Li, J., \& Prigerson, H. G. (2016). Assessment and associated features of prolonged grief disorder among Chinese bereaved individuals. Comprehensive Psychiatry, 66, 9-16.

Li, Y., \& Li, J. (2017). Death with dignity from the Confucian perspective. Theoretical Medicine and Bioethics, 38(1), 63-81. https://doi.org/10.1016/j.comppsych.2015.12.001.

Mak, M. H. J. (2017). Death: Good Death. Encyclopedia of Global Bioethics, 1-9.

Morrison, R. S., \& Peoples, L. (1999). Using focus group methodology in nursing. The Journal of Continuing 
Education in Nursing, 30(2), 62-65. https://doi.org/10.3928/0022-0124-19990301-06

Neimeyer, R. A. (Ed.). (2015). Death anxiety handbook: Research, instrumentation, and application. Taylor \& Francis.

Nienaber, K., \& Goedereis, E. (2015). Death anxiety and education: A comparison among undergraduate and graduate students. Death studies, 39(8), 483-490. https://doi.org/10.1080/07481187.2015.1047057

Noble, K. G., Houston, S. M., Brito, N. H., Bartsch, H., Kan, E., Kuperman, J. M., ... Schork, N. J. (2015). Family income, parental education and brain structure in children and adolescents. Nature Neuroscience, 18(5), 773-778. http://doi.org/10.1038/nn.3983

Parkes, C. M., \& Prigerson, H. G. (2013). Bereavement: Studies of Grief in Adult Life. Routledge.

Parkes, C.M., Laungani, P., \& Young, B. (1997). Death and Bereavement. London: Routledge.

Sandler, I. N., Ayers, T. S., Wolchik, S. A., Tein, J. Y., Kwok, O. M., Haine, R. A., ... Weyer, J. L. (2003). The family bereavement program: Efficacy evaluation of a theory-based prevention program for parentally bereaved children and adolescents. Journal of Consulting and Clinical Psychology, 71(3), 587. http://dx.doi.org/10.1037/0022-006X.71.3.587

Schoenfelder, E. N., Tein, J. Y., Wolchik, S., \& Sandler, I. N. (2015). Effects of the family bereavement program on academic outcomes, educational expectations and job aspirations 6 years later: The mediating role of parenting and youth mental health problems. Journal of Abnormal Child Psychology, 43(2), 229-241. http://doi.org/10.1007/s10802-014-9905-6

Schooling, C. M., Jiang, C., Lam, T. H., Zhang, W., Cheng, K. K., \& Leung, G. M. (2011). Parental death during childhood and adult cardiovascular risk in a developing country: the Guangzhou Biobank Cohort Study. PLoS One, 6(5). https://doi.org/10.1371/journal.pone.0019675

Stroebe, M. S., Hansson, R. O., Stroebe, W., \& Schut, H. (2001). Future Directions for Bereavement Research. In M. S. Stroebe, R. O. Hansson, W. Strobe, \& H. (Eds.), Schut Handbook of Bereavement Research (pp. 741-763). Washington. D.C.: American Psychological Association.

Taku, K., Calhoun, L. G., Cann, A., \& Tedeschi, R. G. (2008). The role of rumination in the coexistence of distress and posttraumatic growth among bereaved Japanese university students. Death Studies, 32(5), 428-444. https://doi.org/10.1080/07481180801974745

Zhang, D. W. (2011). Death attitudes and affect factors of Chinese university students. Journal of Modern Preventive Medicine, 38(4), 675-677 (in Chinese).

Zhang, P., Li, C. Z., Zhao, Y. N., Xing, F. M., Chen, C. X., Tian, X. F., \& Tang, Q. Q. (2016). The mediating role of emotional intelligence between negative life events and psychological distress among nursing students: A cross-sectional study. Nurse Education Today, 44, 121-126. http://doi.org/10.1016/j.nedt.2016.05.025

Zhang, W. W. (2015). Bereavement and grief of Chinese university students (Master thesis). Shanghai Normal University (in Chinese).

\section{Copyrights}

Copyright for this article is retained by the author(s), with first publication rights granted to the journal.

This is an open-access article distributed under the terms and conditions of the Creative Commons Attribution license (http://creativecommons.org/licenses/by/4.0/). 